\title{
Idiopathic laryngotracheal stenosis: Effective definitive treatment with laryngotracheal resection
}

\author{
Simon K. Ashiku, MD* \\ Akin Kuzucu, MD \\ Hermes C. Grillo, MD \\ Cameron D. Wright, MD \\ John C. Wain, MD \\ Bruce Lo, MD \\ Douglas J. Mathisen, MD
}

See related editorial on page 10 .

From the Division of Thoracic Surgery, Massachusetts General Hospital, Boston, Mass.

Read at the Eighty-second Annual Meeting of The American Association for Thoracic Surgery, Washington, DC, May 5-8, 2002.

Received for publication May 20, 2002; revisions requested July 8, 2002; revisions received Nov 5, 2002; accepted for publication Nov 14, 2002.

Address for reprints: Dr Douglas J. Mathisen, Massachusetts General Hospital, Blake 1570, 55 Fruit St, Boston, MA 02214.

*Present address: Department of Surgery, Beth Israel Deaconess Medical Center, Boston, Mass.

J Thorac Cardiovasc Surg 2004;127:99-107 $0022-5223 / \$ 30.00$

Copyright () 2004 by The American Association for Thoracic Surgery

doi:10.1016/j.jtcvs.2002.11.001
Objective: Little was known about idiopathic laryngotracheal stenosis when it was first described. We have operated on 73 patients with idiopathic laryngotracheal stenosis, have confirmed its mode of presentation and response to surgical therapy, and have established long-term follow-up.

Methods: Charts of 73 patients treated surgically for idiopathic laryngotracheal stenosis between 1971 and 2002 were retrospectively reviewed.

Results: All patients were treated with a single-staged laryngotracheal resection, with (36/73) and without (37/73) a posterior membranous tracheal wall flap. Nearly all were women (71/73), with a mean age of 46 years (range, 13-74 years). Twenty-eight (38\%) of 73 had undergone a previous procedure with laser, dilation, tracheostomy, T-tube, or laryngotracheal operations. After laryngotracheal resection, the majority of patients (67/73) were extubated in the operating room, and 7 required temporary tracheostomies, only 1 of whom was among the last 30 patients. All were successfully decannulated. There was no perioperative mortality. Principal morbidity was alteration of voice quality, which was mild and tended to improve with time. Sixty-seven (91\%) of 73 patients had good to excellent long-term results with voice and breathing quality and do not require further intervention for their idiopathic laryngotracheal stenosis.

Conclusion: Idiopathic laryngotracheal stenosis is an entity that occurs almost exclusively in women and is without a known cause. It is not a progressive process, but the timing of the operation is crucial. Single-staged laryngotracheal resection is successful in restoring the airway while preserving voice quality in more than $90 \%$ of patients. Protective tracheostomy is now rarely required (1/30). Long-term follow-up shows a stable airway and improvement in voice quality.

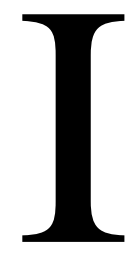

diopathic laryngotracheal stenosis (ILTS) is a rare disease characterized by an inflammatory cicatricial stenosis at the level of the cricoid and upper trachea. Patients experience dyspnea on exertion that progresses to dyspnea at rest, noisy breathing, stridor, or a combination of these symptoms. Symptoms develop over the course of months to years, with patients often given erroneous diagnoses and treated for asthma. It occurs almost exclusively in women in their third, fourth, and fifth decades who have no identifiable cause of airway stenosis. Its cause remains unknown. ${ }^{1}$

Patients are considered to have ILTS if they have an inflammatory cicatricial stenosis of the subglottis, upper trachea, or both and known causes are excluded. Known causes of subglottic stenosis include post-intubation injury; airway trauma, including external injury; inhalational burns and irradiation; specific and nonspecific 
tracheal infections, including bacterial tracheitis, tuberculosis, histoplasmosis, and diphtheria; collagen vascular diseases, including Wegener's granulomatosis, relapsing polychondritis, polyarteritis, scleroderma, and sarcoid; amyloid disease; and congenital causes. ${ }^{1}$

Treatment options range from conservative short-term therapies to definitive surgical procedures. ${ }^{2-4}$ The shortterm therapies that have been described are airway dilation, local corticosteroid injection, and laser ablation. Tracheostomies or airway stents are generally not helpful and only complicate the eventual repair by extending the area of damaged trachea. Definitive surgical procedures that have been used involve either cartilage grafts to enlarge the laryngotracheal lumen or segmental laryngotracheal resection with primary end-to-end anastomosis. However, the optimal management strategy in the treatment of ILTS remains controversial. Its rarity has led to the reporting of only 3 series beyond occasional case reports. ${ }^{1,5,6}$ Authors have reported different outcomes for definitive surgical therapy and disagree over the effectiveness of surgical intervention in providing a cure for ILTS. Some authors consider ILTS to be a relentlessly progressive disease and advocate repeated palliative procedures indefinitely. ${ }^{6}$

In our experience, however, most patients are best treated with definitive segmental resection of the involved airway. The optimal timing of the surgical approach is crucial to the success and safety of the operation. The presence of inflammation or extension of the process close to the vocal cords demands temporizing maneuvers to maintain the airway. Delay in operation is also necessary to allow patients taking corticosteriods to discontinue their use. Not all patients with ILTS are surgical candidates, and the decision when and in whom to operate requires mature surgical judgment. We report short- and long-term results of single-staged laryngotracheal resection in 73 patients.

\section{Materials and Methods Study Design}

Between 1971 and 2002, 73 patients were treated for ILTS with a single-staged laryngotracheal resection. We have reanalyzed our original 35 patients treated with a single-staged laryngotracheal resection, ${ }^{1}$ and to these, we have added our subsequent experience. Their charts were retrospectively reviewed to abstract demographic and clinicopathologic data.

Long-term results were collected from patients by using detailed questionnaires approved by the institutional review board for human studies. The current quality of each patient's breathing and voice and any subsequent airway problems or interventions were noted. Inquires were made into any newly diagnosed conditions, such as gastroesophageal reflux disease (GERD), ${ }^{7,8}$ Wegener's granulomatosis, ${ }^{9}$ or postnasal drip with severe allergic rhinitis. Antineutrophil cytoplasm antibody test results were reviewed. ${ }^{10,11}$ Patients with other known causes of subglottic stenosis were excluded from the study.
Six patients had to be excluded because they were eventually found to have Wegener's granulomatosis. Two male patients who had been given a diagnosis of ILTS were also excluded because detailed review uncovered a history of tracheal tuberculosis in the distant past in one, whereas the other went on to have progressive tracheal and bilateral bronchial stenosis, which is inconsistent with the clinical features and usual course of ILTS.

\section{Operative Treatment}

Endoscopic examination was done to confirm the diagnosis, assess gross characteristics, and measure the extent of laryngotracheal involvement. Examinations were performed after achievement of general inhalation anesthesia by using Jackson rigid ventilating bronchoscopes with magnifying telescopes. Stenoses were dilated to allow examination of the distal airway and subsequent passage of an endotracheal tube. Endoscopy was usually performed at the time of planned surgical resection. In patients in whom inflammation was more severe or extended to just below the vocal cords, dilation was used to allow the process to subside, possibly enabling later resection.

All patients were treated with a single-staged laryngotracheal resection with primary end-to-end anastomotic reconstruction. In most cases the lesion typically involved the subglottic larynx to various degrees on its anterior and lateral lumenal surface at the level of the cricoid plate (Figure 1). The recurrent laryngeal nerves were protected by beveling off the cricoid anteriorly and laterally while preserving the posterior plate. ${ }^{3,12,13}$ The extent of anterior cricoid resection ranged from complete, with a line of transection through the cricothyroid membrane, to none at all, depending on the extent of involvement. Tracheal resection depended on the distal extent of the lesion. The trachea was appropriately tailored so that the proximal trachea coapted well with the cut edge of the larynx (Figure 2, $A$ and $B$ ). Both proximally and distally, 2-0 Vicryl traction sutures (Ethicon, Inc, Somerville, NJ) were placed in the midlateral position. Interrupted 4-0 Vicryl sutures were used to fashion the anastomosis. The midline of the thyroid cartilage was approximated to the midline of the tracheal prow. Then 2-0 Vicryl traction sutures were tied, followed by individual 4-0 Vicryl anastomotic sutures.

This operation was modified in patients in whom the stenosis was circumferential, affecting the mucosa overlying the cricoid plate. Sparing the posterior cricoid plate preserves the recurrent laryngeal nerves. The line of mucosal division was performed high on the posterior cricoid plate to excise involved mucosa and submucosa (Figure 2,C). Mucosal resection stopped short of the superior border of the cricoid plate immediately below the arytenoid cartilages. Subperichondrial resection of cartilage was not necessary. In these patients the rostrum, or prow, of the proximal tracheal cartilage was shaped as described above, but posteriorly, a broad-based flap of membranous wall was fashioned that was advanced to resurface the denuded posterior cricoid plate (Figure $2, C)$. Four sutures were placed through the cartilaginous portion of the inferior margin of the cricoid plate and the outer portion of the membranous wall of the trachea below the proximal edge of the flap to fix the membranous wall to the inferior edge of the cricoid plate (Figure 2, D). The posterior portion of the anastomosis was made with interrupted 4-0 Vicryl sutures placed only through the full thickness of mucosa and submucosa of the pos- 


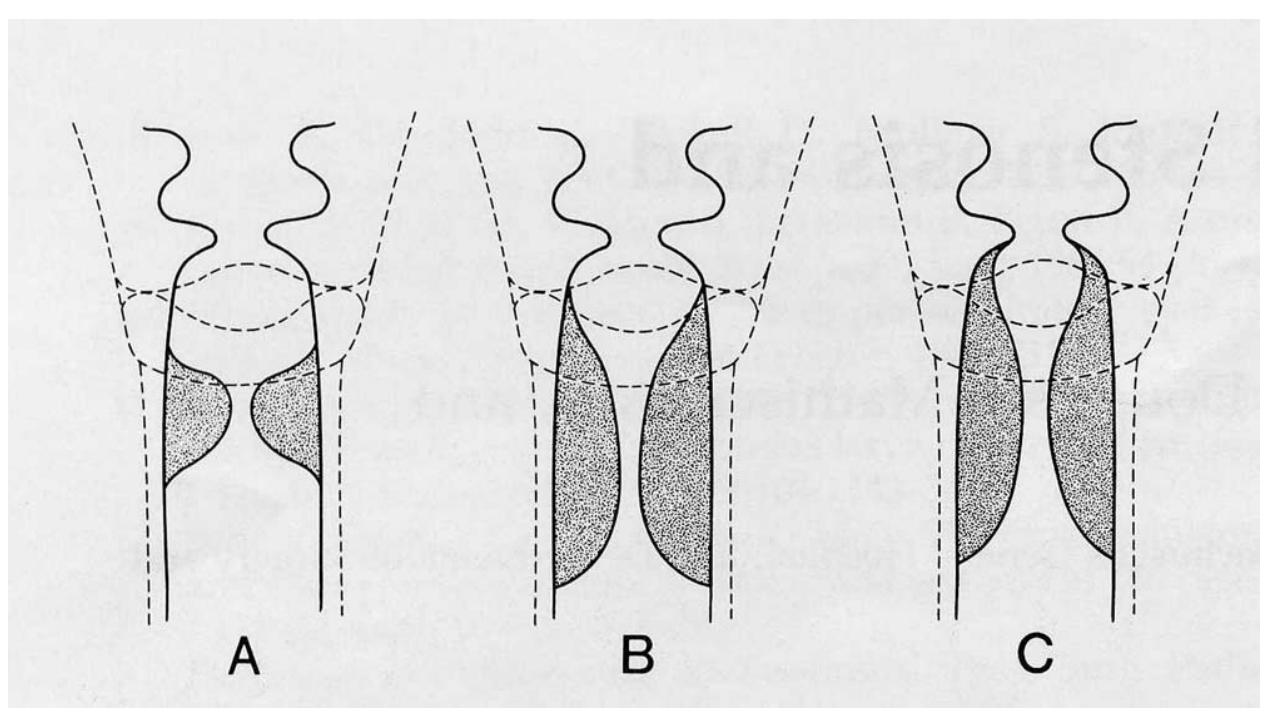

Figure 1. Diagrams of typical distribution of idiopathic tracheal and laryngotracheal stenosis. A, Lesion confined principally to the upper trachea, but which usually impinges on the low subglottic larynx at cricoid level. B, Lesion commencing in the subglottic larynx with narrowing of the immediately subglottic space which, however, leaves an "atrium" beneath the cords. The maximum stenosis is at cricotracheal level. C, More marked stenosis immediately below the vocal cords with little space for anastomosis even at that level. (Ann Thorac Surg 1993;56:80-7 [Figure 1]; published with permission of Edith Tagrin.)

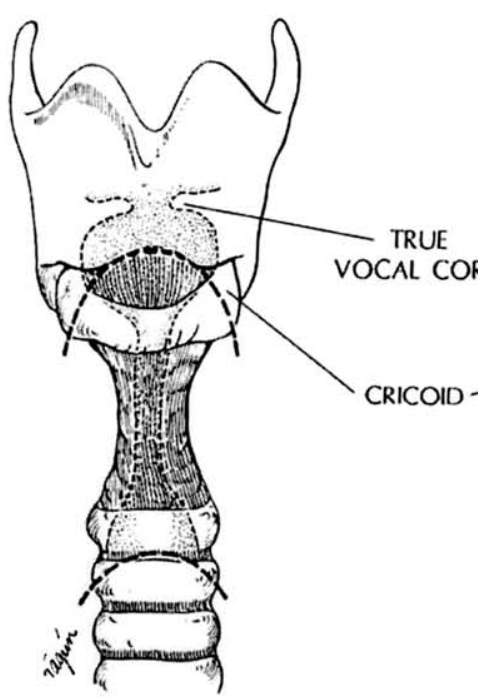

A

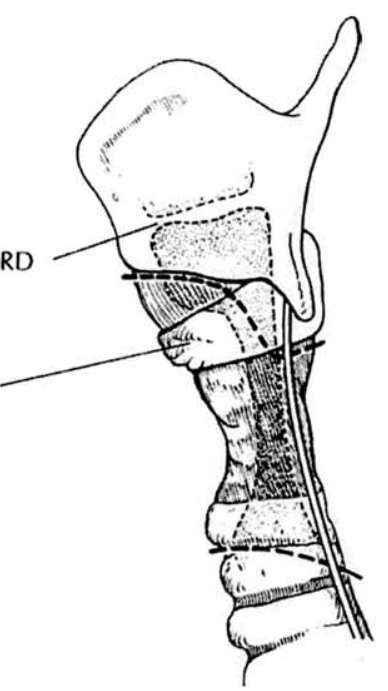

B

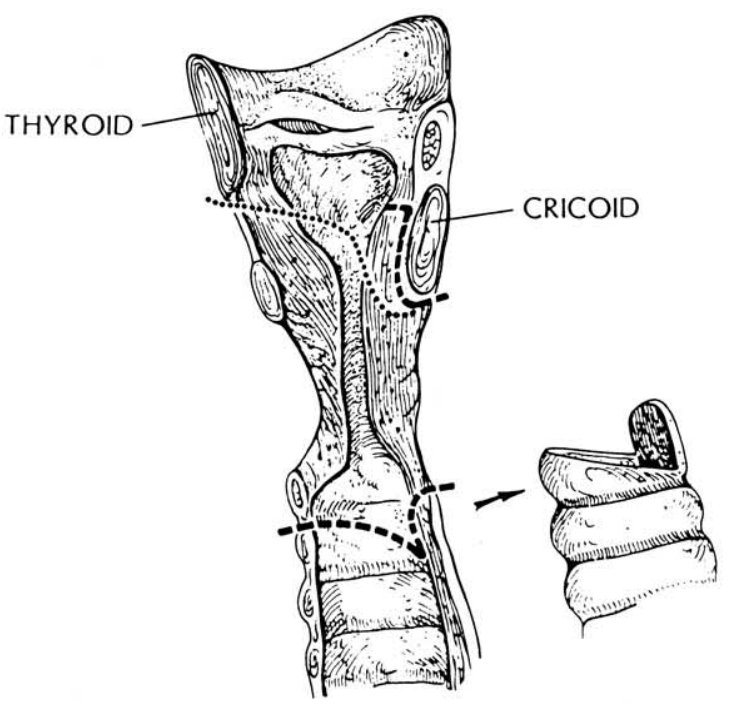

C

Figure 2. Technique of laryngotracheal resection and reconstruction in the presence of circumferential stenosis. A, External lines of anterior division of larynx and trachea are indicated by dashed lines. B, Lateral view indicates removal of anterior arch of cricoid but conservation of posterolateral laminae and posterior cricoid to preserve recurrent laryngeal nerves. A single proximal intact cartilage of trachea is beveled as shown. $\mathrm{C}$, The posterior stenosis is excised from the front of the posterior cricoid plate, preserving the plate and its posterior perichondrium. A broadly based flap of membranous tracheal wall is fashioned distally, as shown, to resurface the cricoid plate. (Ann Thorac Surg 1982;33:3-8 [Figures 7 and 8]. Published with permission of Edith Tagrin.)

terior wall of the larynx and then through the full thickness of the membranous wall of the trachea, which was inverted so that the suture knots lay external to the lumen (Figure 2,E). When the lesion extended proximally toward the conus elasticus, it was necessary to accept some residual narrowing because of the high level of the anastomosis. 


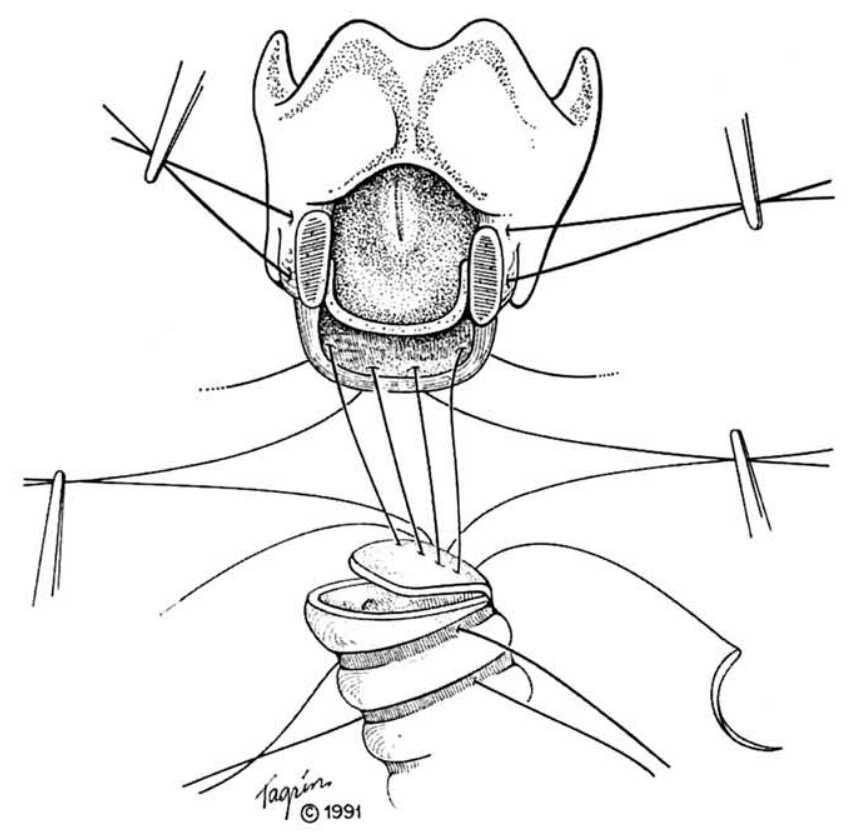

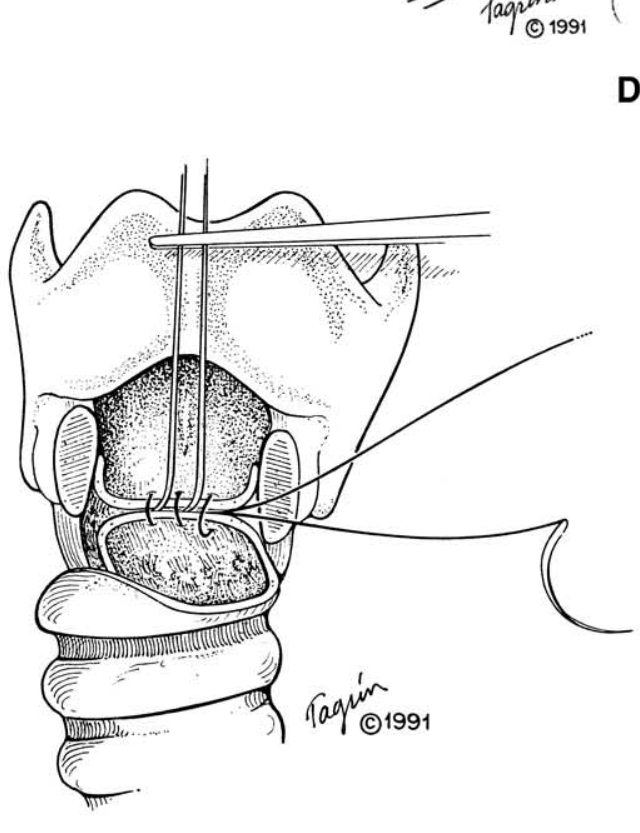

$\mathbf{E}$

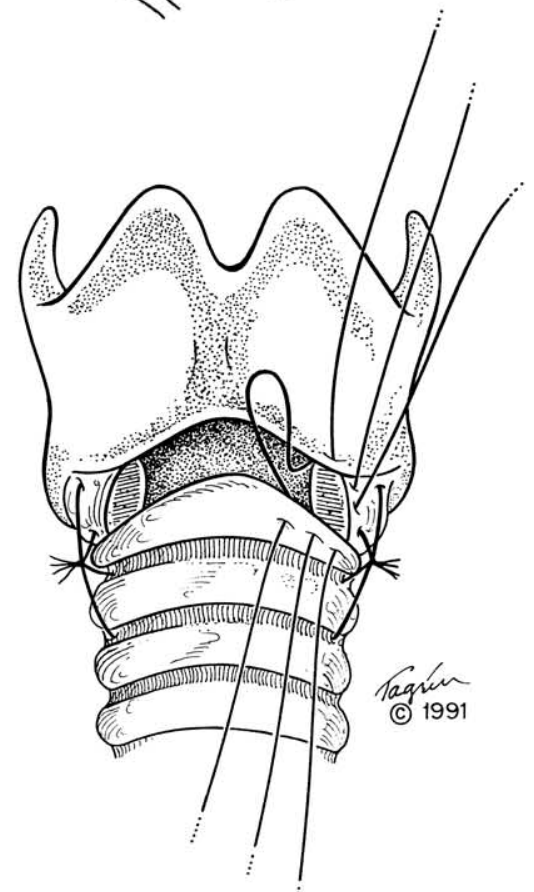

$\mathbf{F}$

Figure 2. Cont'd. D, The base of the posterior flap is tied to the inferior margin of cricoid plate. Lateral traction sutures are also shown. E, The flap is sutured into place. The knots are posterior to the mucosa. F, After placement of all posterior and posterolateral anastomotic sutures, the stay sutures are tied, then the external posterior sutures, followed by the posterolateral anastomotic sutures, and then the internal posterior mucosal sutures. Anterior and anterolateral sutures are then placed and tied, completing the anastomosis. (Ann Thorac Surg 1992;53:54-63 [Figure 10]; published with permission of Edith Tagrin.)

All resections were performed through a cervical collar incision. One patient required a suprahyoid laryngeal release because of the extent of airway resection. ${ }^{14}$ Before extubation, patients underwent bronchoscopy to clear the distal airways of secretions and blood. A chin stitch was placed to remind patients to maintain cervical flexion. Maneuvers to limit postoperative laryngeal edema included minimizing fluids, elevating the head of the bed, cautioning patients against using their voices, and administering racemic epinephrine. Patients were observed for at least one evening in the intensive care unit. Anastomosis was routinely evaluated by means 
of bronchoscopy or laminar tracheal tomograms on the sixth or seventh day. If the patency and integrity of the anastomosis was satisfactory, the protecting chin stitch was removed, and the patient was discharged.

\section{Long-term Follow-up}

Results were characterized as "excellent," "good," "fair," "poor," and "failure." A result was considered excellent if voice (including singing voice) and respiration were both considered normal by the patient. A good result was judged to be only a mild change in vocal characteristics. This usually meant some degree of weakness in the ability to project the voice and an inability to sing as well as preoperatively. Mild dyspnea on major exertion was accepted as a good result because the maximum airway that could be obtained in such cases was the anatomically narrower diameter of the immediate subglottic airway. A result was considered fair if there was vocal cord hoarseness or an intermittently weak voice or exercise tolerance was limited by postoperative airway stenosis requiring occasional dilations. Exercise tolerance was limited by the diameter of the airway, and occasional dilation was still required. A poor result was a very hoarse or nearly whispered voice or dyspnea from upper airway obstruction on ordinary activity. Also included was the need for frequent and continued airway dilations. Failure indicated the need for a permanent tracheostomy or $\mathrm{T}$ tube.

\section{Results}

\section{Presentation}

All but 2 patients were female. The average age at operation was 46 years, with a range of 13 to 74 years. The presenting symptom was dyspnea on exertion in $52 \%$ of patients and noisy breathing-stridor in $48 \%$. Twenty-three patients were incorrectly given the initial diagnosis of asthma, and many were treated for years before laryngotracheal stenosis was diagnosed. Duration of symptoms before presentation ranged from a few months to 32 years, with an average of 4 years. Fifty-four patients had a previous history of brief intubation for unrelated surgical procedures for an average of 1.7 intubations per individual, whereas 19 patients had no prior history of intubation. Fifteen patients had either existing GERD at the time of presentation or had symptoms at some point postoperatively. We do not think GERD was responsible for the symptoms in any of these patients with ILTS. If such a relationship existed in this patient group, one would have expected to see vocal cord inflammation at the time of the operation or progression of disease postoperatively. Neither was seen. Sixteen patients reported having severe allergies with postnasal drip. Six patients were subsequently found to have Wegener's granulomatosis and were excluded from the study.

\section{Prior Treatment}

Before referral, 28 patients had undergone at least one prior invasive procedure elsewhere. These included a total of at least 29 dilations, 31 laser ablations, 6 tracheostomies, 6
T-tube placements or stents, 2 laryngeal enlargement operations, and 1 tracheal resection and reconstruction.

\section{Extent and Nature of Lesion and Resection}

The cephalad extent of the lesions ranged from just below the cords to the first tracheal ring. Most stenoses began at the cricoid. The point of maximal stenosis was almost always located in the region from the upper edge of the cricoid to the lower edge of the first tracheal ring. The majority of lesions were circumferential, although occasionally eccentric, and extended from 0.5 to $5.0 \mathrm{~cm}$ in length. The majority were about $2 \mathrm{~cm}$ long.

The extent of surgical resection ranged from 1 to $5 \mathrm{~cm}$, with an average of $2.6 \mathrm{~cm}$. The superior extent of the laryngeal resection was the cricothyroid membrane in 59 patients, and partial resection of the anterior cricoid was required in 10 patients. No cricoid resection was necessary in 4 patients. Posterior mucosal involvement required mucosal resection and advancement of a posterior membranous wall flap in 36 patients.

\section{Short-term Results}

Sixty-seven patients were extubated in the operating room and required no further airway intervention during their hospital stay. Five patients had protecting tracheostomies placed at the completion of the operation, as did 2 patients at 5 and 10 days postoperatively. This allowed time for the resolution of glottic and laryngeal edema occasioned by the high surgical procedure. These remained for a median of 16 days, with a range of 7 to 90 days. Protective tracheostomies were performed in the early years of the study, and only one has been used in the last 30 patients. One patient was left intubated postoperatively as an alternative to tracheostomy. She was extubated on the sixth postoperative day. One postoperative wound infection required incision and drainage. One patient had postoperative cervical subcutaneous emphysema; the results of wound re-exploration was negative. One patient had a small pneumothorax, which resolved. One patient initially had impaired swallowing but a normal swallowing evaluation. Two patients had late swallowing problems, both of which were resolved with a single dilation. The intensive care unit stay averaged 1.3 days, with a range of 1 to 6 days. The average length of stay was 8.5 days, with a range of 5 to 18 days. There were no deaths.

In the early follow-up period, 6 patients required dilation for obstructing granulation tissue. One patient had laser ablation of granulation tissue elsewhere. The number of treatments ranged from 1 to 2 .

\section{Long-term Results}

Follow-up to the present period was completed in 68 patients. Four additional patients had long-term follow-up, but it did not extend to the current time period. One patient from 
South America was lost to follow-up. The median length of follow-up was 8.0 years, with an average of 7.9 years. Excellent results were obtained in $19(26 \%)$ of 72 patients. Good results were obtained in $47(64 \%)$ of 72 patients. Inability to project voice and change in singing voice was the major complaint in these patients. Fair results occurred in $5(7 \%)$ of 72 patients. The most common problems were noisy breathing and shortness of breath with moderate exertion and the occasional need for dilation. A poor result was seen in $1(1.3 \%)$ patient, who has continued to require at least yearly dilations. A few issues regarding this patient deserve mention. First, she had been treated with radioactive iodine for hyperthyroidism. Second, her lesion extended from the lower cricoid for $5 \mathrm{~cm}$ into the distal trachea with small damaged rings. These findings suggest a different diagnosis. Finally, she had inflammation extending into the subglottic space at the time of the operation. There were no failures.

\section{Discussion}

ILTS remains a rare disease with an unknown cause. Unlike postintubation tracheal stenosis, which remains fixed and does not extend to previously uninjured trachea, the natural history of these lesions was not known, and their future course could not be predicted initially. ${ }^{15}$ Such unknown lesions could possibly progress or regress on their own accord. Additionally, many of these patients had severe inflammation accompanying the lesions, which often commenced immediately below the vocal cords. The surgical approach would therefore leave partially involved tissue at the site, with unknown consequences for future recurrence. The uncertainties have led to controversies regarding the optimal management strategy for ILTS and also to an initially conservative approach on our part.

In 1993, Grillo and colleagues ${ }^{1}$ reported a series in which a single-staged laryngotracheal resection was performed in 35 patients, with good to excellent outcome in $91 \%$. A fair result was obtained in 2 patients who required annual dilation, and there was one failure in a patient who required permanent tracheostomy. This male patient went on to have progressive tracheal and bilateral bronchial stenosis, which is inconsistent with the clinical features and usual course of ILTS. Hence he was excluded from the current study. Of 14 other patients, 5 were seen in consultation only and treated elsewhere, 5 were treated with laryngeal enlargement procedures elsewhere, and 4 were managed conservatively with dilations at varying but generally long intervals. Of the patients treated conservatively, 2 underwent successful operations after years of annual dilations, 1 has declined an operation and continues to be dilated at yearly intervals, and 1 was later given a diagnosis of Wegener's granulomatosis.

Dedo and Catten ${ }^{6}$ believed that ILTS is a progressive disease that cannot be cured and hence advocated repeated palliative procedures indefinitely. Fifty-two patients with ILTS were described. Seven were treated with resection, and all had restenosis. Forty-three other patients were treated with endoscopic laser submucosal resection and mucosal flap rotation with mixed results, most requiring continued procedures or tracheostomies. Of the 50 patients reported on, 17 still require tracheostomies, 15 have required more than 10 procedures each, and 21 seemed to be "apparently disease-free over the long-term." Overall, the median number of procedures per patient was 6 , with an average of 8 .

Park and coworkers ${ }^{5}$ suggested that 2 groups of patients exist with ILTS, those with a mild form that can be either observed or that requires only 1 or 2 dilations and those with more complex recurring disease who require resection. In 10 patients with ILTS, conservative therapies failed in 4, and of these, 2 were treated with single-staged laryngotracheal resection with excellent results. Two were being considered for surgical intervention. Four had good results with just 1 to 2 laser incisions and dilations with follow-up from 1 to 7 years. Two required no treatment.

In our experience ILTS is a disease that does not progress after surgical resection. Good-to-excellent results were obtained with definitive segmental resection in more than $90 \%$ of patients. These patients were able to return to a normal life without need for repeated and perpetual airway intervention. Despite partial laryngeal resection, they showed only mild changes in voice characteristics, such as a slight deepening of the voice, loss of singing voice, and diminished projection. These complaints occurred in 42 of the 67 patients in the good-to-excellent categories. Noisy breathing associated with mild shortness of breath on major exertion was less of a problem. Although diminished voice projection was troublesome for some patients in both business meetings and social engagements, they were uniformly pleased with the overall outcome.

ILTS has now become one of the most common indications for laryngotracheal resection at the Massachusetts General Hospital. We believe that most patients are best treated with definitive segmental resection of the involved airway and primary reconstruction in a single stage. However, the optimal timing of the surgical approach is crucial to the success and safety of the operation and requires mature surgical judgment. The presence of active inflammation extending to just below the cords or involving the cords demands temporizing maneuvers to maintain the airway until the process resolves. Some patients have required repeated dilations while allowing time for the inflammatory process to abate before a surgical resection was successfully undertaken. Operating in an inflamed field places the patient's airway at risk in the immediate postoperative period and might contribute to a less desirable long-term outcome. Delay in operation is also necessary to allow patients taking 
corticosteriods to discontinue their use. Corticosteriods can impair healing of the anastomosis and lead to airway complications.

The known causes of subglottic stenosis that should not be treated with surgical resection should be excluded before surgical intervention. These include collagen vascular diseases, such as Wegener's granulomatosis, relapsing polychondritis, polyarteritis, scleroderma, and sarcoid. ${ }^{1}$ Wegener's granulomatosis was later diagnosed in 6 of our patients who had undergone resection for what was thought to be ILTS. These patients were not cured and required continued treatment. Preoperative antineutrophil cytoplasm antibody tests should be performed in all patients to screen for Wegener's granulomatosis. Rhuematology consultation is helpful in interpreting results and excluding other collagen vascular diseases.

Others have implicated GERD as a cause of ILTS. ${ }^{7,8}$ In this study 15 patients had either existing GERD at the time of presentation or had symptoms at some point postoperatively. GERD does not appear to have been a factor in these patients because none had associated vocal cord inflammation, and only 2 required a subsequent dilation for mild restenosis. If such a causal relationship existed, one would have expected to see vocal cord inflammation at the time of the operation or progression of disease postoperatively. This was not observed. Furthermore, it is difficult to reconcile why only women would be affected given the equal prevalence of GERD in men. Nevertheless, all patients with ILTS should be carefully evaluated for GERD.

As experience has grown, we eliminated the earlier practice of placing a protective tracheostomy at the time of the operation. Tracheostomies can complicate the anastomotic healing and are best avoided. We have not electively placed one at the time of surgical intervention in the last 30 cases, although one patient required placement at day 5. Maneuvers to limit postoperative laryngeal edema include minimizing fluids, elevating the head of the bed, and administering racemic epinephrine to prevent laryngeal edema. Rarely, an especially high laryngotracheal resection will cause enough laryngeal edema to necessitate 1 or 2 doses of corticosteriods to avoid reintubation, tracheostomy, or both. Heliox, with its low viscosity, is sometimes useful in these circumstances to gain enough time for the other maneuvers to take effect. The patient is cautioned against speaking during this period because it might contribute to the laryngeal edema. Cervical flexion is maintained for 5 to 7 days, after which time the patient is advised not to extend the neck forcefully for another week. Before removing the chin-tochest suture, we routinely examine the anastomosis with a flexible bronchoscope or obtain tracheal tomograms to ensure normal healing.

Although ILTS is a rare disease, the rising awareness in the medical community of its existence and of the availabil- ity of definitive treatment has led to increased experience with its management. We have found that ILTS is not a progressive process and that most patients do not go on to have a primary disease that can be linked to the airway obstruction. Single-staged segmental laryngotracheal resection effectively cures most patients when optimally timed and undertaken in experienced centers. Dilation might be justifiable as an initial treatment to assess response. Repeated dilations are reserved for patients in whom definitive resection is inappropriate by reason of florid inflammation or proximity of stenosis to the vocal cords.

\section{References}

1. Grillo HC, Mark EJ, Mathisen DJ, Wain JC. Idiopathic Laryngotracheal stenosis and its management. Ann Thorac Surg. 1993;56: 80-7.

2. McCaffrey TV. Management of subglottic stenosis in the adult. Ann Otol Rhinol Laryngol. 1991;100:90-4.

3. Grillo HC. Primary reconstruction of airway after resection of subglottic laryngeal and upper tracheal stenosis. Ann Thorac Surg. 1982; 33:3-18.

4. Jacobson I, Benjamin B, Eckstein R. Idiopathic subglottic stenosis: diagnosis and endoscopic laser treatment. Ann Otol Rhinol Laryngol. 1997;106:770-4.

5. Park SS, Streitz JM, Rebeiz EE, Shapshay SM. Idiopathic subglottic stenosis. Arch Otolaryngol Head Neck Surg. 1995;121:894-7.

6. Dedo HH, Catten MD. Idiopathic progressive subglottic stenosis: finding and treatment in 52 patients. Ann Otol Rhinol Laryngol. 2000;110:305-11.

7. Walner DL, Stern Y, Gerber ME, Rudolph C, Baldwin CY, Cotton RT. Gastroesophageal reflux in patients with subglottic stenosis. Arch Otolaryngol Head Neck Surg. 1998;124:551-5.

8. Koufman JA. The otolaryngologic manifestations of gastroesophageal reflux disease (GERD): a clinical investigation of 225 patients using ambulatory 24-hour $\mathrm{pH}$ monitoring and an experimental investigation of the role of acid and pepsin in the development of laryngeal injury. Laryngoscope. 1991;101:1-64.

9. Langford CA, Sneller MC, Hallahan CW, Hoffman GS, Kammerer WA, Talar-Williams C, et al. Clinical features and therapeutic management of subglottic stenosis in patients with Wegener's granulomatosis. Arthritis Rheum. 1996;39:1754-60.

10. Specks U, Wheatley CL, McDonald TJ, Rohrbach MS, DeRemee RA. Anticytoplasmic autoantibodies in the diagnosis and follow-up of Wegener's granulomatosis. Mayo Clin Proc. 1989;64:28-36.

11. De Vries N, Gans RO, Donker AM, Goldschmeding R, Hoorntje SJ, Snow GB. Autoantibodies against constituents of neutrophils in the diagnosis and treatment of (isolated) subglottic stenosis. Arch Otolaryngol Head Neck Surg. 1992;118:1120-3.

12. Grillo HC, Mathisen DJ, Wain JC. Laryngotracheal resection and reconstruction for subglottic stenosis. Ann Thorac Surg. 1992;53:54-63.

13. Pearson FG, Brito-Filomeno L, Cooper JD. Experience with partial cricoid resection and thyrotracheal anastomosis. Ann Otol Rhinol Laryngol. 1986;95:582-5.

14. Montgomery WW. Suprahyoid release for tracheal anastomosis. Arch Otolaryngol. 1974;99:255-60.

15. Grillo HC, Donahue DM. Post intubation tracheal stenosis. Semin Thorac Cardiovasc Surg. 1996;8:370-80.

\section{Discussion}

Dr F. Griffith Pearson (Mansfield, Ontario, Canada). ILTS is a rare condition, as you pointed out. As a referral center in Toronto, we have had a similar experience, although smaller, and it began in the early 1970 s when we first saw a few cases. We seem to have seen this odd disease with increasing frequency since that time. 
I have not had the opportunity to review our Toronto experience in detail, but I will not be far off accuracy in saying that we have had about 20 patients. Only one was male. Again, this was a group of patients in which those that I have seen have been women, with the youngest being in their early 20 s. There is a patient in this series who is 13 . I have not seen a young teenager with the condition. You said it is a disease of young women, and the mean age in your article is 46 years, and that will please a lot of 46-year-olds.

The pathology that we have seen is similar. I mean, it is just nonspecific inflammation that occurs within the cricoid and then might involve a certain amount of trachea below, usually not much in our experience. However, for the adjacent inflammation in the mucosa, because the primary lesion is inflammatory with some type of really superficial ulceration, probably in the mucous membrane itself, when that ultimately heals, it heals with scarring, and you end up with a mature lesion. But on occasion, fortunately not often, it might lie within a few millimeters of the inferior margin of the vocal fold.

The principles of management, as you pointed out, are the same as for all benign inflammatory lesions: you want to wait until the inflammation is as diminished as possible. The operation we have done is similar. There are some differences in the way we manage the posterior cricoid plate. We tend to use stainless-steel wire, very fine wire, with the knots inside for the posterior wall, but those are only minor technical issues that do not really change the outcome.

The results in this reported series are indeed excellent-90\% good-to-excellent results - and you have had no reoperations. I can say that we have had no mortality. We have had no reoperations. We have had only a few patients who required interval dilation thereafter.

You mentioned Wegener's granulomatosis, which you excluded. You had 6 patients listed as having Wegener's granulomatosis who were excluded. We had 2 patients who had resections for what we thought was idiopathic subglottic stenosis. One was the only male patient in the series, a young man who, 10 years later, presented with quite classical Wegener's granulomatosis, and I have no doubt that that is what the subglottic lesion was. The other was a young woman who, 12 years later, had the same experience. Of interest is that their Wegener's granulomatosis involved their lungs systemically, but it did not impair the result in the subglottic region. It is a very small number of cases, but it is nevertheless of interest should you ever bump into that situation.

"Excellent" is classified in this presentation as a normal voice. It has been my experience that if you are within, I would say, 3 or $5 \mathrm{~mm}$ of the inferior margins of the cords, that you never have a perfectly normal voice for all practical purposes. You did, however, have a group of patients who you categorized as having A-level damage, in which the damage lay at the bottom of the cricoid cartilage or a little below. Do you know whether this was the group that ended up with an excellent outcome as far as voice is concerned?

The hardest thing here is to determine how long to wait and how you are going to get rid of the acute inflammation when you see some of these cases acutely. I know we have certainly waited for up to a year or longer in an occasional patient. Can you tell me how long some of these patients might have been delayed with interval dilation before they were operated on? And you did mention the exhibition of steroids. I think you said maybe steroid injection, and I wonder if you can enlarge on that aspect of management. Thank you.

Dr Ashiku. I thank Dr Pearson for his insightful comments. I know that he has had a lot of experience in this area.

We did exclude 6 patients with Wegener's granulomatosis. These patients probably had Wegener's granulomatosis at the time of the operation; they went on to show signs of the disease later on. I think this group of patients did not do well ultimately. We do not have complete and accurate follow-up on them, but we do know relatively well how they did. Four patients went on to require repeated dilatations at frequent intervals and probably do not have a good result from the operation. Two patients had fair to good results and have not required any further therapy yet, but they are relatively early in their course of care.

In terms of excellent results, you are correct. Whenever you resect the anterior wall of the cricoid, you do change the ability of the patient to alter their pitch. I think actually in sex-change operations, where they want to change the voice of a patient, they actually sew the anterior wall of the cricoid to the thyroid cartilage, and this gives them a deeper voice. I think that did happen in a large percentage of our patients. Essentially, we placed a graft of cartilage by pulling up our trachea onto that area, and this resulted in deepening of voices.

You are correct that those patients who only had a tracheal resection, those 4 patients, and patients who had partial resections of the cricoid did fall into the excellent category, and the higher up the resection, the higher the risk of voice abnormalities. The number one complaint in the bulk of patients, and this was the reason that these patients all fell into the "good" category, was based on voice changes. Patients complained that they did not have their singing voice anymore, that they could not project in a crowd, and for some people this was troublesome in their meetings, business meetings, or in social engagements.

As for how long to wait for inflammation to resolve, that is not something that I looked at when I went through all the charts. I did not analyze how many patients were dilated and how long we waited for them. That is something I am thinking about going back and analyzing. It is an excellent question. My sense of that, and maybe Dr Mathisen might want to comment on that, is that there is a fair number of patients in whom, when we first see the inflammation, the inflammation is extensive enough that we dilate them and wait a period of time, on the order of months, and do rebronchoscopy, and from my limited experience with it, I think that it is usually over the course of months to a year before they ultimately end up requiring treatment. There were a few of the original patients from Dr Grillo's original report who were treated conservatively with dilatations. The bulk of those patients ultimately crossed over into surgical intervention, some up to 10 or 15 years later.

Dr Douglas E. Wood (Seattle, Wash). Dr Ashiku, that was a very nicely presented paper and great results. My congratulations for both.

I just have one question. One of the postulates for the potential cause of idiopathic tracheal stenosis is silent reflux. I am wondering if you have investigated that in any of your patients. Does that become a part of your investigation, to exclude reflux disease as a potential cause? How routine is that in your workup? 
Dr Ashiku. In terms of our article, we did go back and look at how many patients had symptoms of gastroesophageal reflux at the time of their presentation. We also inquired whether patients had any reflux subsequent to that. It is actually a fairly small percentage. I do not recall the number at this moment, but it was around 19 or so patients who had symptoms of severe esophagitis, but the remainder of the patients said they had no symptoms whatsoever. Of course, as a retrospective study, we did not have any objective evidence of it. In terms of working up our patients for gastroesophageal reflux, I think in our practice it is usually screening for it in terms of questioning. We do not use any $\mathrm{pH}$ monitoring to obtain objective data on it. 